\title{
Hyers-Ulam-Rassias Stability of Some Additive Fuzzy Set-Valued Functional Equations with the Fixed Point Alternative
}

\author{
Yonghong Shen, ${ }^{1,2}$ Yaoyao Lan, ${ }^{3}$ and Wei Chen ${ }^{4}$ \\ ${ }^{1}$ School of Mathematics and Statistics, Tianshui Normal University, Tianshui 741001, China \\ ${ }^{2}$ School of Mathematics and Statistics, Beijing Institute of Technology, Beijing 100081, China \\ ${ }^{3}$ Department of Mathematics and Finance, Chongqing University of Arts and Sciences, Yongchuan 402160, China \\ ${ }^{4}$ School of Information, Capital University of Economics and Business, Beijing 100070, China
}

Correspondence should be addressed to Yonghong Shen; shenyonghong2008@hotmail.com

Received 2 December 2013; Accepted 23 January 2014; Published 5 March 2014

Academic Editor: Geraldo Botelho

Copyright (C) 2014 Yonghong Shen et al. This is an open access article distributed under the Creative Commons Attribution License, which permits unrestricted use, distribution, and reproduction in any medium, provided the original work is properly cited.

Let $Y$ be a real separable Banach space and let $\left(\mathscr{K}_{C}(Y), d_{\infty}\right)$ be the subspace of all normal fuzzy convex and upper semicontinuous fuzzy sets of $Y$ equipped with the supremum metric $d_{\infty}$. In this paper, we introduce several types of additive fuzzy set-valued functional equations in $\left(\mathscr{K}_{C}(Y), d_{\infty}\right)$. Using the fixed point technique, we discuss the Hyers-Ulam-Rassias stability of three types additive fuzzy set-valued functional equations, that is, the generalized Cauchy type, the Jensen type, and the Cauchy-Jensen type additive fuzzy set-valued functional equations. Our results can be regarded as important extensions of stability results corresponding to single-valued functional equations and set-valued functional equations, respectively.

\section{Introduction}

In 1940, Ulam [1] proposed the following question concerning the stability of group homomorphisms.

Let $G_{1}$ be a group and let $G_{2}$ be a metric group with the metric $d(\cdot, \cdot)$. Given $\epsilon>0$, does there exist a $\delta>0$ such that if a function $h: G_{1} \rightarrow G_{2}$ satisfies the inequality $d(h(x y), h(x) h(y))<\delta$ for all $x, y \in G_{1}$, then there is a homomorphism $H: G_{1} \rightarrow G_{2}$ with $d(h(x), H(x))<\epsilon$ for all $x \in G_{1}$ ?

Afterwards, Hyers [2] gave a first affirmative partial answer to the question of Ulam for Banach spaces. Later, the result of Hyers was generalized by Aoki [3] (for some historical comments regarding the work of Aoki, see [4]) for additive mappings and by Rassias [5] for linear mappings in which the Cauchy difference is allowed to be unbounded. However, the paper of Rassias [5] has provided a lot of influence in the development of what we call Hyer-Ulam stability or HyersUlam-Rassias stability of functional equations. Hereafter, a generalization of Rassias's Theorem obtained by Găvruţa [6] by replacing the Cauchy difference with a more general majorant function in the spirit of Rassias' approach. Until now, the stability problems for different types of functional equations in various spaces have been extensively studied. For more detail, the reader can refer to [7]. Among the studies of these problems, it is worth mentioning that Radu [8] discarded the direct method which was frequently used and proposed a novel method to establish the stability of Cauchy functional equation via fixed point technique. Recently, Ciepliński [9] summarized some applications of several types of fixed point theorems to the Hyers-Ulam stability of functional equations. As of now, this method has been successfully used in the study of stability problems of many types of functional equations in abstract spaces.

As defined in [7], let $E_{1}$ and $E_{2}$ be two appropriate spaces, where $E_{2}$ is equipped with the metric $d$. For some $p, q \in$ $\mathbb{N}(\mathbb{N}$ denotes the set of all natural numbers), the following functions

$$
\begin{gathered}
g_{i}: E_{1}^{q} \longrightarrow E_{1} \quad(i \in 1,2, \ldots, p), \\
G: E_{2}^{p} \times E_{1}^{q} \longrightarrow E_{2}
\end{gathered}
$$


are given. Assume that $\varphi, \Phi: E_{1}^{q} \rightarrow[0, \infty)$ are functions satisfying some given conditions. If for every function $f$ : $E_{1} \rightarrow E_{2}$ satisfying the inequality

$$
\begin{aligned}
& d\left(G \left(f\left(g_{1}\left(x_{1}, x_{2}, \ldots, x_{q}\right)\right), f\left(g_{2}\left(x_{1}, x_{2}, \ldots, x_{q}\right)\right), \ldots\right.\right. \\
& \left.\left.\quad f\left(g_{p}\left(x_{1}, x_{2}, \ldots, x_{q}\right)\right), x_{1}, x_{2}, \ldots, x_{p}\right), 0\right) \\
& \leq \varphi\left(x_{1}, x_{2}, \ldots, x_{q}\right)
\end{aligned}
$$

for all $x_{1}, x_{2}, \ldots, x_{q} \in E_{1}^{q}$, there exists a function $F: E_{1} \rightarrow E_{2}$ such that

$$
\begin{gathered}
G\left(F\left(g_{1}\left(x_{1}, x_{2}, \ldots, x_{q}\right)\right), F\left(g_{2}\left(x_{1}, x_{2}, \ldots, x_{q}\right)\right), \ldots,\right. \\
\left.F\left(g_{p}\left(x_{1}, x_{2}, \ldots, x_{q}\right)\right), x_{1}, x_{2}, \ldots, x_{p}\right)=0
\end{gathered}
$$

for all $x_{1}, x_{2}, \ldots, x_{q} \in E_{1}^{q}$ and

$$
d(f(x), F(x)) \leq \Phi(x, x, \ldots, x)
$$

for all $x \in E_{1}$, then we say that the functional equation

$$
\begin{gathered}
G\left(f\left(g_{1}\left(x_{1}, x_{2}, \ldots, x_{q}\right)\right), f\left(g_{2}\left(x_{1}, x_{2}, \ldots, x_{q}\right)\right), \ldots,\right. \\
\left.f\left(g_{p}\left(x_{1}, x_{2}, \ldots, x_{q}\right)\right), x_{1}, x_{2}, \ldots, x_{p}\right)=0
\end{gathered}
$$

has the Hyers-Ulam-Rassias stability or the above functional equation is stable in the sense of Hyers-Ulam-Rassias. In particularly, if the functions $\varphi$ and $\Phi$ are replaced by two constants, $\delta$ and $K \delta(K>0)$, respectively, then we say that the functional equation has the Hyers-Ulam stability or it is stable in the sense of Hyers-Ulam.

In 2008, Mirmostafaee and Moslehian [10] initiated the study of stability problems of functional equations in fuzzy setting. Specifically, they considered the stability of the Cauchy functional equation in a fuzzy normed space. In the same year, they together with Mirzavaziri [11] proved the stability of the Jensen functional equation in the same space. Since then, the fuzzy stability problems of various types of functional equations have been extensively investigated by different authors $[12,13]$. At the same time, the fixed point method has been widely used to prove the fuzzy stability of several types of functional equations $[14,15]$.

In summary, one can see that the (fuzzy) stability for a single-valued functional equation is whether, for a given mapping satisfying almost a functional equation (which means that the mapping is close to a solution of the functional equation), there exists an exact solution of the functional equation which can be used to approximate the given mapping. Typically, a metric associated with the corresponding space is chosen to characterize the functional inequality. In 2009, Nikodem and Popa [16] considered the general solution of set-valued maps satisfying linear inclusion relation, which can be regarded as a generalization of the additive single-valued functional equation. By means of the inclusion relation, Lu and Park [17] first investigated the stability of two types of additive set-valued functional equations. In the following, Park et al. [18] further studied the stability problems of the quadratic, cubic, and quartic set-valued functional equations in a similar way. However, it should be pointed out that, in their studies, the inclusion relation is applied to characterize the set-valued functional inequality rather than an appropriate metric. Recently, similar to the method that is used to deal with the single-valued functional equations, Kenary et al. [19] proved the stability of several types of set-valued functional equations via the fixed point approach, in which the Hausdorff metric is adopted to characterize the set-valued functional inequality.

The purpose of this paper is to extend the set-valued functional equations to fuzzy set-valued functional equations and establish some stability results of several important fuzzy set-valued functional equations, including the generalized Cauchy, Jensen, and Cauchy-Jensen type fuzzy set-valued functional equations. Notice that the supremum metric, as a generalization of the Hausdorff metric, is applied to characterize the fuzzy set-valued functional inequality. More importantly, the corresponding single-valued and set-valued functional equations acted as special cases will be included in our results.

\section{Preliminaries}

In what follows, we begin with some related concepts and fundamental results, which are mainly derived from [20-23]. Let $\mathbb{R}, \mathbb{R}^{+}$, and $\mathbb{R}^{n}$ denote the set of all real numbers, the set of all nonnegative real numbers, and the $n$-dimensional Euclidean space, respectively.

Let $Y$ be a real separable Banach space with the norm $\|\cdot\|_{Y}$. We denote by $\mathscr{K}(Y)$ and $\mathscr{K}_{C}(Y)$ the set of all nonempty compact subsets of $Y$ and the set of all nonempty compact convex subsets of $Y$, respectively.

Let $A$ and $B$ be two nonempty subsets of $Y$ and let $\lambda \in \mathbb{R}$. The (Minkowski) addition and scalar multiplication can be defined by

$$
\begin{gathered}
A+B=\{a+b \mid a \in A, b \in B\}, \\
\lambda A=\{\lambda a \mid a \in A\} .
\end{gathered}
$$

Notice that the sets $\mathscr{K}(Y)$ and $\mathscr{K}_{C}(Y)$ are closed under the operations of addition and scalar multiplication. In fact, these two operations induce a linear structure on $\mathscr{K}(Y)$ and $\mathscr{K}_{C}(Y)$ with zero element $\{0\}$, respectively. It should be noted that this linear structure is just a cone rather than a vector space because, in general, $A+(-1) A \neq\{0\}$. Moreover, for all $\lambda, \mu \in \mathbb{R}$, it follows that

$$
\lambda(A+B)=\lambda A+\lambda B, \quad(\lambda+\mu) A \subseteq \lambda A+\mu A .
$$

In particular, if $A$ is convex and $\lambda \mu \geq 0$, then $(\lambda+\mu) A=$ $\lambda A+\mu A$.

Furthermore, we can define the Hausdorff separation of $B$ from $A$ by

$$
d_{H}^{*}(B, A)=\inf \left\{\epsilon>0 \mid B \subseteq A+\epsilon \overline{S_{1}}\right\},
$$

where $\overline{S_{1}}$ denotes the closed unit ball in $Y$; that is, $\overline{S_{1}}=\{y \in$ $\left.Y \mid\|y\|_{Y} \leq 1\right\}$. Meantime, the Hausdorff separation of $A$ from $B$ can also be defined in a similar way. 
Based on these two types of separations, the Hausdorff distance between nonempty subsets $A$ and $B$ is defined by

$$
d_{H}(A, B)=\max \left\{d_{H}^{*}(A, B), d_{H}^{*}(B, A)\right\} \text {. }
$$

In general, if $A, B \in \mathscr{K}(Y)$ or $\mathscr{K}_{C}(Y)$, then $d_{H}(\lambda A, \lambda B)=$ $|\lambda| d_{H}(A, B)$ for all $\lambda \in \mathbb{R}$. In addition, according to some of the properties of Hausdorff distance, if we restrict our attention to the nonemtpy closed subsets $\mathscr{C}(Y)$ of $Y$, then it can be verified that $\left(\mathscr{C}(Y), d_{H}\right)$ is a metric space. In fact, it follows from [20] that $\left(\mathscr{C}(Y), d_{H}\right)$ is a complete metric space. Clearly, $\mathscr{K}(Y)$ and $\mathscr{K}_{C}(Y)$ are closed subsets of $\mathscr{C}(Y)$. Hence, $\left(\mathscr{K}(Y), d_{H}\right)$ and $\left(\mathscr{K}_{C}(Y), d_{H}\right)$ are also complete metric spaces.

In 1991, Inoue [22] introduced the concept of Banach space valued fuzzy sets in order to extend the usual fuzzy sets defined on $\mathbb{R}$ or $\mathbb{R}^{n}$. In other words, the base space of a fuzzy set is replaced by a more general Banach space.

For a given real separable Banach space $Y$, a fuzzy set defined on $Y$ is a mapping $u: Y \rightarrow[0,1]$. Denote by $\mathscr{F}(Y)$ the set of all fuzzy sets defined on $Y$. Let $\mathscr{F}_{K}(Y)$ denote the class of fuzzy sets $u: Y \rightarrow[0,1]$ with the following properties:

(i) $u$ is normal, that is, $[u]^{1}=\{y \in Y \mid u(y) \geq 1\}$ is nonempty;

(ii) $u$ is upper semicontinuous;

(iii) $[u]^{\alpha}=\{y \in Y \mid u(y) \geq \alpha\}$ is compact for each $\alpha \epsilon$ $(0,1]$

(iv) $[u]^{0}=\overline{\bigcup_{\alpha \in(0,1]}[u]^{\alpha}}$ is a bound subset of $Y$.

Notice that the conditions (ii) and (iv) imply that $[u]^{0}$ is also compact. Moreover, we use the notation $\mathscr{F}_{K C}(Y)$ to denote the subspace of $\mathscr{F}(Y)$ whose members also satisfy

(v) $u$ is fuzzy convex; that is, $[u]^{\alpha}$ is convex for each $\alpha \epsilon$ $(0,1]$.

A linear structure can be defined in $\mathscr{F}(Y)$ in a similar way to fuzzy sets in $\mathbb{R}$ or $\mathbb{R}^{n}$ by

$$
\begin{gathered}
(u \oplus v)(y)=\sup _{x+z=y} \min \{u(x), v(z)\}, \\
(\gamma u)(y)= \begin{cases}u\left(\frac{y}{\gamma}\right), & \text { if } \gamma \neq 0, \\
I_{0}(y), & \text { if } \gamma=0,\end{cases}
\end{gathered}
$$

for $u, v \in \mathscr{F}(Y)$ and $\gamma \in \mathbb{R}$, where $I_{0}(y)=0$ if $y \neq 0$ and $I_{0}(0)=1$. Then $\mathscr{F}(Y)$ is closed under these operations and level setwise

$$
[u \oplus v]^{\alpha}=[u]^{\alpha}+[v]^{\alpha}, \quad[\lambda u]^{\alpha}=\lambda[u]^{\alpha}
$$

for each $\alpha \in[0,1]$ and $\lambda \in \mathbb{R}$. Similar to the closeness of $\mathscr{K}_{C}(Y)$, it is easy to know that $\mathscr{F}_{K C}(Y)$ is also closed under these operations. Based on the statement mentioned above, we can easily obtain the following lemma.
Lemma 1. For any $u, v \in \mathscr{F}_{K C}(Y)$ and $\lambda, \mu \in \mathbb{R}$, the following equalities hold:

(i) $\lambda(u \oplus v)=\lambda u \oplus \lambda v$;

(ii) $\lambda(\mu u)=(\lambda \mu) u$;

(iii) $(\lambda+\mu) u=\lambda u \oplus \mu u$ for any $\lambda, \mu \geq 0$.

Remark 2. The Lemma 1 shows that $\mathscr{F}_{K C}(Y)$ is just a cone defined on $Y$ rather than a vector space.

As a generalization of the Hausdorff metric $d_{H}$ in $\mathscr{K}(Y)$, we will define the supremum metric $d_{\infty}$ in $\mathscr{F}_{C}(Y)$. For $u, v \in$ $\mathscr{F}_{C}(Y)$, the supremum metric is defined by

$$
d_{\infty}(u, v)=\sup _{\alpha \in(0,1]} d_{H}\left([u]^{\alpha},[v]^{\alpha}\right) .
$$

Remark 3. Every ordinary crisp subset $A$ of $Y$ can be identified with the fuzzy set on $Y$ by its characteristic function $\chi_{A}: Y \rightarrow\{0,1\}$, that is, with $\chi_{A}(y)=1$ if $y \in A$ and $\chi_{A}(y)=0$ if $y \notin A$. Therefore, if $A \in \mathscr{K}(Y)$ (or $A \in \mathscr{K}_{C}(Y)$ ), then $\chi_{A} \in \mathscr{F}_{C}(Y)$ (or $\left.\mathscr{F}_{K C}(Y)\right)$, and vice versa.

From Remark 3, for any $A, B \in \mathscr{K}(Y)\left(\right.$ or $\left.\mathscr{F}_{K C}(Y)\right)$, it follows that

$$
d_{\infty}\left(\chi_{A}, \chi_{B}\right)=\sup _{\alpha \in(0,1]} d_{H}\left(\left[\chi_{A}\right]^{\alpha},\left[\chi_{B}\right]^{\alpha}\right)=d_{H}(A, B) .
$$

In particular, if $A$ and $B$ degenerate into two singleton sets $\{a\}$ and $\{b\}$, then we can infer from equality (13) that $d_{\infty}\left(\chi_{\{a\}}, \chi_{\{b\}}\right)=d(a, b)$, where $d$ denotes the usual metric between $a$ and $b$.

In view of the property of the Hausdorff metric, it is easy to see that $d_{\infty}(\lambda u, \lambda v)=\lambda d_{\infty}(u, v)$ for any $\lambda \geq 0$. Restricting attention to the set $\mathscr{F}_{K C}(Y)$, we can prove that $\left(\mathscr{F}_{K C}(Y), d_{\infty}\right)$ is a complete metric space by the method analogous to that used in [21] (see Proposition 7.2.3). theory.

Finally, we quote a fundamental result in fixed point

Theorem 4 (Diaz and Margolis [24]). Let $(X, d)$ be a complete generalized metric space, that is, one for which d may assume infinite values. Suppose that $J: X \rightarrow X$ is a strictly contractive mapping with Lipschitz constant $L<1$. Then, for each given element $x \in X$, either

$$
d\left(J^{n} x, J^{n+1} x\right)=\infty
$$

for all $n \geq 0$ or there exists an $n_{0} \in \mathbb{N}$ such that

(i) $d\left(J^{n} x, J^{n+1} x\right)<\infty$ for all $n \geq n_{0}$;

(ii) the sequence $\left\{J^{n} x\right\}$ converges to a fixed point $y^{*}$ of $J$;

(iii) $y^{*}$ is the unique fixed point of $J$ in the set $Y=\{y \in X \mid$ $\left.d\left(J^{n_{0}} x, y\right)<\infty\right\}$

(iv) $d\left(y, y^{*}\right) \leq(1 /(1-L)) d(y, J y)$ for all $y \in Y$. 


\section{Stability of the Generalized \\ Cauchy Type Additive Fuzzy Set-Valued Functional Equation}

In this section, we will establish the Hyers-Ulam-Rassias stability of the generalized Cauchy type additive fuzzy setvalued functional equation by employing the fixed point method.

Definition 5. Let $X$ be a cone with the vertex 0 and let $f: X \rightarrow \mathscr{F}_{K C}(Y)$ be a fuzzy set-valued mapping. The generalized Cauchy type additive fuzzy set-valued functional equation is defined by

$$
f(\xi x+\eta y)=\xi f(x) \oplus \eta f(y)
$$

for all $x, y \in X$ and for some $\xi, \eta>0$ with $\xi+\eta \neq 1$.

Especially, if $\xi=\eta=1$, then (15) is called the standard Cauchy type additive fuzzy set-valued functional equation. Every solution of (15) is called a generalized Cauchy type additive fuzzy set-valued mapping.

Example 6. Let $X=\mathbb{R}^{+}$and $Y=\mathbb{R}$. Suppose that $f: \mathbb{R}^{+} \rightarrow$ $\mathscr{F}_{K C}(\mathbb{R})$ is a triangular fuzzy set-valued mapping, that is, for every $t \in \mathbb{R}^{+}, f(t)$ is a triangular fuzzy number in $\mathbb{R}$, which is defined by

$$
f(t)=(t-a t, t, t+b t), \quad t \in \mathbb{R}^{+},
$$

where $a$ and $b$ are two nonnegative real numbers. By the definition of $\alpha$-level set, we can obtain that

$$
[f(t)]^{\alpha}=[t-a t(1-\alpha), t+b t(1-\alpha)]
$$

for every $t \in \mathbb{R}^{+}$. Then, for every $\alpha \in[0,1]$, it is easy to verify that

$$
[f(\xi x+\eta y)]^{\alpha}=\xi[f(x)]^{\alpha}+\eta[f(y)]^{\alpha}
$$

for all $x, y \in \mathbb{R}^{+}$and $\xi, \eta>0$ with $\xi+\eta \neq 1$. That is, $f$ is a solution of (15) in $\mathbb{R}^{+}$.

Remark 7. A triangular fuzzy number $u \in \mathscr{F}_{K C}(\mathbb{R})$ is characterized by an ordered triple $\left(x_{l}, x_{c}, x_{r}\right) \in \mathbb{R}^{3}$ with $x_{l} \leq x_{c} \leq x_{r}$ such that the support set $[u]^{0}=\left[x_{l}, x_{r}\right]$ and 1-level set $[u]^{1}=\left\{x_{c}\right\}$.

Remark 8. More generally, if $X=\mathbb{R}^{+}$, by Lemma 1, it is easy to see that $f(t)=t u_{0}$ is a solution of (15) for any $t \in \mathbb{R}^{+}$and any fixed $u_{0} \in \mathscr{F}_{K C}(Y)$.

Theorem 9. Let $\varphi: X \times X \rightarrow[0,+\infty)$ be a function such that there exists a positive constant $L<1$ satisfying

$$
\varphi(x, y) \leq \frac{L}{\xi+\eta} \varphi((\xi+\eta) x,(\xi+\eta) y)
$$

for all $x, y \in X$ and for some $\xi, \eta>0$ with $\xi+\eta \neq 1$. Suppose that $f: X \rightarrow \mathscr{F}_{\mathrm{KC}}(Y)$ is a mapping satisfying

$$
d_{\infty}(f(\xi x+\eta y), \xi f(x) \oplus \eta f(y)) \leq \varphi(x, y)
$$

for all $x, y \in X$. Then

$$
A(x)=\lim _{n \rightarrow \infty}(\xi+\eta)^{n} f\left(\frac{x}{(\xi+\eta)^{n}}\right)
$$

exists for each $x \in X$ and defines a unique generalized Cauchy type additive fuzzy set-valued mapping $A: X \rightarrow \mathscr{F}_{K C}(Y)$ such that

$$
d_{\infty}(f(x), A(x)) \leq \frac{L}{(\xi+\eta)(1-L)} \varphi(x, x)
$$

for all $x \in X$.

Proof. Replacing $x=y$ in (20), by Lemma 1, we get

$$
d_{\infty}(f((\xi+\eta) x),(\xi+\eta) f(x)) \leq \varphi(x, x)
$$

for all $x \in X$. Thus, we can obtain

$$
\begin{aligned}
& d_{\infty}\left(f(x),(\xi+\eta) f\left(\frac{x}{\xi+\eta}\right)\right) \\
& \quad \leq \varphi\left(\frac{x}{\xi+\eta}, \frac{x}{\xi+\eta}\right) \leq \frac{L}{\xi+\eta} \varphi(x, x)
\end{aligned}
$$

for all $x \in X$.

Consider the set $E=\left\{g \mid g: X \rightarrow \mathscr{F}_{K C}(Y), g(0)=I_{0}\right\}$ and introduce the generalized metric $D$ on $E$, which is defined by

$$
\begin{gathered}
D(g, h)=\inf \left\{\mu \in(0, \infty) \mid d_{\infty}(g(x), h(x))\right. \\
\leq \mu \varphi(x, x), \forall x \in X\}
\end{gathered}
$$

where, as usual, inf $\emptyset=\infty$. In can easily be verified that $(E, D)$ is a complete generalized metric space (see [25], Theorem 2.4).

Now, we consider the linear mapping $J: E \rightarrow E$ such that

$$
J g(x)=(\xi+\eta) g\left(\frac{x}{\xi+\eta}\right)
$$

for all $x \in X$.

Let $g, h$ be given such that $D(g, h) \leq \epsilon$. Then

$$
d_{\infty}(g(x), h(x)) \leq \epsilon \varphi(x, x)
$$

for all $x \in X$. Hence, we have

$$
\begin{aligned}
d_{\infty}(J g(x), J h(x)) \\
\quad=d_{\infty}\left((\xi+\eta) g\left(\frac{x}{\xi+\eta}\right),(\xi+\eta) h\left(\frac{x}{\xi+\eta}\right)\right) \\
\quad=(\xi+\eta) d_{\infty}\left(g\left(\frac{x}{\xi+\eta}\right), h\left(\frac{x}{\xi+\eta}\right)\right) \\
\leq \epsilon(\xi+\eta) \varphi\left(\frac{x}{\xi+\eta}, \frac{x}{\xi+\eta}\right) \\
\leq \frac{L}{\xi+\eta} \epsilon(\xi+\eta) \varphi(x, x)=\operatorname{L\epsilon } \varphi(x, x)
\end{aligned}
$$


for all $x \in X$. So $D(g, h) \leq \epsilon$ implies that $D(J g, J h) \leq L \epsilon$. This means that

$$
D(J g, J h) \leq L D(g, h)
$$

for all $g, h \in E$. Evidently, $J$ is a strictly contractive selfmapping on $E$ with the Lipschitz constant $L<1$.

Moreover, it follows from $(24)$ that $D(f, J f) \leq L /(\xi+\eta)$. According to Theorem 4, there exists a mapping $A: X \rightarrow$ $\mathscr{F}_{c}(Y)$ satisfying the following.

(i) $A$ is a fixed point of $J$ : that is,

$$
\frac{1}{\xi+\eta} A(x)=A\left(\frac{x}{\xi+\eta}\right)
$$

for all $x \in X$. The mapping $A$ is the unique fixed point of $J$ in the set

$$
M=\{g \in E \mid D(f, g)<\infty\},
$$

which implies that $A$ is the unique mapping satisfying (30) such that there exists a $r \in(0,1)$ satisfying

$$
d_{\infty}(f(x), A(x)) \leq r \varphi(x, x)
$$

for all $x \in X$.

(ii) $D\left(J^{n} f, A\right) \rightarrow 0$ as $n \rightarrow \infty$. This implies the equality

$$
\lim _{n \rightarrow \infty}(\xi+\eta)^{n} f\left(\frac{x}{(\alpha+\beta)^{n}}\right)=A(x)
$$

for all $x \in X$.

(iii) $D(f, A) \leq(1 /(1-L)) D(f, J f)$, which implies the inequality

$$
D(f, A) \leq \frac{1}{1-L} \cdot \frac{L}{\xi+\eta}=\frac{L}{(\xi+\eta)(1-L)} .
$$

This implies that the inequality (22) holds.

By (20), we can obtain that

$$
\begin{aligned}
& d_{\infty}\left((\xi+\eta)^{n} f\left(\xi \frac{x}{(\xi+\eta)^{n}}+\eta \frac{x}{(\xi+\eta)^{n}}\right)\right. \\
& \left.\quad(\xi+\eta)^{n}\left(\xi f\left(\frac{x}{(\xi+\eta)^{n}}\right) \oplus \eta f\left(\frac{y}{(\xi+\eta)^{n}}\right)\right)\right) \\
& \leq(\xi+\eta)^{n} \varphi\left(\frac{x}{(\xi+\eta)^{n}}, \frac{y}{(\xi+\eta)^{n}}\right) \\
& \leq(\xi+\eta)^{n} \cdot \frac{L^{n}}{(\xi+\eta)^{n}} \varphi(x, x)=L^{n} \varphi(x, y),
\end{aligned}
$$

which tends to zero as $n \rightarrow \infty$ for all $x, y \in X$. Thus,

$$
A(\xi x+\eta y)=\xi A(x) \oplus \eta A(y)
$$

for all $x, y \in X$ and therefore the mapping $A: X \rightarrow$ $\mathscr{F}_{K C}(Y)$ is a generalized Cauchy type additive fuzzy setvalued mapping as desired.
Corollary 10. Let $X$ be a cone with the vertex 0 contained in a real normed space and let $p, \theta$ be positive real numbers with $p>1$ (resp. $0<p<1$ ). Suppose that $f: X \rightarrow \mathscr{F}_{K C}(Y)$ is a mapping satisfying

$$
d_{\infty}(f(\xi x+\eta y), \xi f(x) \oplus \eta f(y)) \leq \theta\left(\|x\|^{p}+\|y\|^{p}\right)
$$

for all $x, y \in X$ and for some $\xi, \eta>0$ with $\xi+\eta>1$ (resp. $<1)$. Then

$$
A(x)=\lim _{n \rightarrow \infty}(\xi+\eta)^{n} f\left(\frac{x}{(\xi+\eta)^{n}}\right)
$$

exists for each $x \in X$ and defines a unique generalized Cauchy type additive fuzzy set-valued mapping $A: X \rightarrow \mathscr{F}_{K C}(Y)$ such that

$$
d_{\infty}(f(x), A(x)) \leq \frac{2 \theta\|x\|^{p}}{(\xi+\eta)^{p}-(\xi+\eta)}
$$

for all $x \in X$.

Proof. In Theorem 9, let $\varphi(x, y)=\theta\left(\|x\|^{p}+\|x\|^{p}\right)$. Then we can choose $L=(\xi+\eta)^{1-p}$ and we get the desired result.

Corollary 11. Let $X$ be a cone with the vertex 0 contained in a real normed space and let $p, \theta$ be positive real numbers with $p>1 / 2$ (resp. $p<1 / 2$ ). Suppose that $f: X \rightarrow \mathscr{F}_{K C}(Y)$ is a mapping satisfying

$$
d_{\infty}(f(\xi x+\eta y), \xi f(x) \oplus \eta f(y)) \leq \theta\|x\|^{p}\|y\|^{p}
$$

for all $x, y \in X$ and for some $\xi, \eta>0$ with $\xi+\eta>1$ (resp. $<1$ ). Then

$$
A(x)=\lim _{n \rightarrow \infty}(\xi+\eta)^{n} f\left(\frac{x}{(\xi+\eta)^{n}}\right)
$$

exists for each $x \in X$ and defines a unique generalized Cauchy type additive fuzzy set-valued mapping $A: X \rightarrow \mathscr{F}_{K C}(Y)$ such that

$$
d_{\infty}(f(x), A(x)) \leq \frac{\theta\|x\|^{2 p}}{(\xi+\eta)^{2 p}-(\xi+\eta)}
$$

for all $x \in X$.

Proof. In Theorem 9, let $\varphi(x, y)=\theta\|x\|^{p}\|y\|^{p}$. Then we can choose $L=(\xi+\eta)^{1-2 p}$ and we get the desired result.

Corollary 12. Let $X$ be a cone with the vertex 0 contained in a real normed space and let $p, q, \theta$ be positive real numbers with $p+q>1$ (resp. $p+q<1)$. Suppose that $f: X \rightarrow \mathscr{F}_{K C}(Y)$ is a mapping satisfying

$$
\begin{aligned}
d_{\infty}( & f(\xi x+\eta y), \xi f(x) \oplus \eta f(y)) \\
& \leq \theta\left(\|x\|^{p}\|y\|^{q}+\|x\|^{p+q}+\|y\|^{p+q}\right)
\end{aligned}
$$

for all $x, y \in X$ and for some $\xi, \eta>0$ with $\xi+\eta>1($ resp. $<1)$. Then

$$
A(x)=\lim _{n \rightarrow \infty}(\xi+\eta)^{n} f\left(\frac{x}{(\xi+\eta)^{n}}\right)
$$


exists for each $x \in X$ and defines a unique generalized Cauchy type additive fuzzy set-valued mapping $A: X \rightarrow \mathscr{F}_{K C}(Y)$ such that

$$
d_{\infty}(f(x), A(x)) \leq \frac{3 \theta\|x\|^{p+q}}{(\xi+\eta)^{p+q}-(\xi+\eta)}
$$

for all $x \in X$.

Proof. In Theorem 9, let $\varphi(x, y)=\theta\left(\|x\|^{p}\|y\|^{q}+\|x\|^{p+q}+\right.$ $\left.\|y\|^{p+q}\right)$. Then we can choose $L=(\xi+\eta)^{1-(p+q)}$ and we get the desired result.

Remark 13. According to the equality (13), all results obtained in this section still hold true, even if the fuzzy set-valued mapping $f: X \rightarrow \mathscr{F}_{K C}(Y)$ degenerates into a set-valued mapping $f: X \rightarrow \mathscr{K}_{C}(Y)$ or a single-valued mapping $f: X \rightarrow Y$. Therefore, these results can be regarded as an important extension of the stability results of the classical additive Cauchy type functional equations and the additive Cauchy type set-valued functional equations. In essence, Theorem 9 extends some main results obtained by Gajda [26], Hyers [2], and Rassias [5].

\section{Stability of the Jensen Type Additive Fuzzy Set-Valued Functional Equation}

In this section, we will prove the Hyers-Ulam-Rassias stability of the Jensen type additive fuzzy set-valued functional equation by using the same method as employed in the previous section.

Definition 14. Let $X$ be a cone with the vertex 0 and let $f$ : $X \rightarrow \mathscr{F}_{K C}(Y)$ be a fuzzy set-valued mapping. The Jensen type additive fuzzy set-valued functional equation is defined by

$$
2 f\left(\frac{x+y}{2}\right)=f(x) \oplus f(y)
$$

for all $x, y \in X$. Every solution of (46) is called a Jensen type additive fuzzy set-valued mapping.

Remark 15. Obviously, it can be checked that the triangular fuzzy set-valued mapping $f$ as defined in Example 6 is also a solution of (46) in $\mathbb{R}^{+}$. Similarly, $f(t)=t u_{0}$ is a solution of (46) for any $t \in \mathbb{R}^{+}$and any fixed $u_{0} \in \mathscr{F}_{K C}(Y)$.

Theorem 16. Let $j \in\{-1,1\}$ be fixed and let $\varphi: X \times X \rightarrow$ $[0,+\infty)$ be a function such that there exists a positive constant $L<1$ satisfying

$$
\varphi(x, 0) \leq 2^{j} L \varphi\left(2^{-j} x, 0\right)
$$

for all $x \in X$. Moreover, assume that $\varphi$ satisfies

$$
\lim _{n \rightarrow \infty} 2^{-j n} \varphi\left(2^{j n} x, 2^{j n} y\right)=0
$$

for all $x, y \in X$. If a mapping $f: X \rightarrow \mathscr{F}_{K C}(Y)$ satisfies $f(0)=I_{0}$ and the inequality

$$
d_{\infty}\left(2 f\left(\frac{x+y}{2}\right), f(x) \oplus f(y)\right) \leq \varphi(x, y)
$$

for all $x, y \in X$, then

$$
A(x)=\lim _{n \rightarrow \infty} 2^{-j n} f\left(2^{j n} x\right)
$$

exists for each $x \in X$ and defines a unique Jensen type additive fuzzy set-valued mapping $A: X \rightarrow \mathscr{F}_{K C}(Y)$ such that

$$
d_{\infty}(f(x), A(x)) \leq \begin{cases}\frac{1}{1-L} \varphi(x, 0), & \text { if } j=-1, \\ \frac{L}{1-L} \varphi(x, 0), & \text { if } j=1\end{cases}
$$

for all $x \in X$.

Proof. Letting $y=0$ in (49). Since $u \oplus I_{0}=u$ for any $u \in$ $\mathscr{F}_{K C}(Y)$, we get

$$
d_{\infty}\left(2 f\left(\frac{x}{2}\right), f(x)\right) \leq \varphi(x, 0)
$$

for all $x \in X$. Furthermore, it follows from (47) that

$$
d_{\infty}\left(f(x), \frac{1}{2} f(2 x)\right) \leq \frac{1}{2} \varphi(2 x, 0) \leq L \varphi(x, 0)
$$

for all $x \in X$.

Consider the set $E=\left\{g \mid g: X \rightarrow \mathscr{F}_{K C}(Y), g(0)=I_{0}\right\}$ and introduce the generalized metric $D$ on $E$, which is defined by

$$
\begin{gathered}
D(g, h)=\inf \left\{\mu \in(0, \infty) \mid d_{\infty}(g(x), h(x))\right. \\
\leq \mu \varphi(x, 0), \forall x \in X\}
\end{gathered}
$$

where, as usual, inf $\emptyset=\infty$. In can easily be verified that $(E, D)$ is a complete generalized metric space (see [25], Theorem 2.4).

We now define the linear mapping $J: E \rightarrow E$ by

$$
J g(x)=2^{-j} g\left(2^{j} x\right)
$$

for all $x \in X$.

Moreover, we can infer from (52) and (53) that

$$
D(f, J f) \leq \begin{cases}1, & \text { if } j=-1, \\ L, & \text { if } j=1 .\end{cases}
$$

The rest of the proof is similar to the proof of Theorem 9.

Corollary 17. Let $j \in\{-1,1\}$ be fixed and let $p, \theta$ be positive real numbers with $p \neq 1$, and let $X$ be a cone with the vertex 0 contained in a real normed space. Suppose that $f: X \rightarrow$ $\mathscr{F}_{K C}(Y)$ is a mapping satisfying

$$
d_{\infty}\left(2 f\left(\frac{x+y}{2}\right), f(x) \oplus f(y)\right) \leq \theta\left(\|x\|^{p}+\|y\|^{p}\right)
$$

for all $x, y \in X$. Then

$$
A(x)=\lim _{n \rightarrow \infty} 2^{-j n} f\left(2^{j n} x\right)
$$


exists for each $x \in X$ and defines a unique Jensen type additive fuzzy set-valued mapping $A: X \rightarrow \mathscr{F}_{K C}(Y)$ such that

$$
d_{\infty}(f(x), A(x)) \leq \begin{cases}\frac{\theta\|x\|^{p}}{1-2^{1-p}}, & \text { if } j=-1, p>1, \\ \frac{\theta\|x\|^{p}}{2^{1-p}-1}, & \text { if } j=1,0<p<1\end{cases}
$$

for all $x \in X$.

Proof. In Theorem 16, let $\varphi(x, y)=\theta\left(\|x\|^{p}+\|y\|^{p}\right)$. Then we can choose $L=2^{j(p-1)}$ and hence we can obtain the desired result.

Remark 18. Theorem 16 and Corollary 17 can be viewed as a direct extension of the stability results of the single-valued Jensen functional equation obtained by Cădariu and Radu [27] and Jung [28], respectively.

\section{Stability of the Cauchy-Jensen Type Additive Fuzzy Set-Valued Functional Equation}

As a combination of the Cauchy and Jensen functional equations, in this section, we will prove that the Hyers-UlamRassias stability of the Cauchy-Jensen type additive fuzzy setvalued functional equation in a similar way as shown before.

Definition 19. Let $X$ be a cone with the vertex 0 and let $f$ : $X \rightarrow \mathscr{F}_{K C}(Y)$ be a fuzzy set-valued mapping. The CauchyJensen type additive fuzzy set-valued functional equation is defined by

$$
\begin{gathered}
f\left(\frac{x+y}{2}+z\right) \oplus f\left(\frac{x+z}{2}+y\right) \oplus f\left(\frac{y+z}{2}+x\right) \\
=2(f(x) \oplus f(y) \oplus f(z))
\end{gathered}
$$

for all $x, y, z \in X$. Every solution of (60) is called a CauchyJensen type additive fuzzy set-valued mapping.

Remark 20. It is easy to see that the Example 6 is also suitable for (60). Similarly, $f(t)=t u_{0}$ is a solution of (60) for any $t \in \mathbb{R}^{+}$and any fixed $u_{0} \in \mathscr{F}_{K C}(Y)$.

Theorem 21. Let $j \in\{-1,1\}$ be fixed and let $\phi: X^{3} \rightarrow$ $[0,+\infty)$ be a function such that there exists a positive constant $L<1$ satisfying

$$
\varphi(x, y, z) \leq 2^{j} L \varphi\left(2^{-j} x, 2^{-j} y, 2^{-j} z\right)
$$

for all $x, y, z \in X$. Assume that $f: X \rightarrow \mathscr{F}_{K C}(Y)$ is a mapping satisfying

$$
\begin{gathered}
d_{\infty}\left(f\left(\frac{x+y}{2}+z\right) \oplus f\left(\frac{x+z}{2}+y\right) \oplus f\left(\frac{y+z}{2}+x\right),\right. \\
2(f(x) \oplus f(y) \oplus f(z))) \leq \phi(x, y, z)
\end{gathered}
$$

for all $x, y, z \in X$. Then

$$
A(x)=\lim _{n \rightarrow \infty} 2^{-j n} f\left(2^{j n} x\right)
$$

exists for each $x \in X$ and defines a unique Cauchy-Jensen type additive fuzzy set-valued mapping $A: X \rightarrow \mathscr{F}_{K C}(Y)$ such that

$$
d_{\infty}(f(x), A(x)) \leq \begin{cases}\frac{L}{6(1-L)} \varphi(x, x, x), & \text { if } j=-1, \\ \frac{1}{6(1-L)} \varphi(x, x, x), & \text { if } j=1\end{cases}
$$

for all $x \in X$.

Proof. Letting $x=y=z$ in (62). By Lemma 1, we can get

$$
d_{\infty}\left(\frac{1}{2} f(2 x), f(x)\right) \leq \frac{1}{6} \phi(x, x, x)
$$

for all $x \in X$. Furthermore, it follows from (61) that

$$
d_{\infty}\left(f(x), 2 f\left(\frac{x}{2}\right)\right) \leq \frac{1}{3} \phi\left(\frac{x}{2}, \frac{x}{2}, \frac{x}{2}\right) \leq \frac{L}{6} \phi(x, x, x)
$$

for all $x \in X$.

Consider the set $E=\left\{g \mid g: X \rightarrow \mathscr{F}_{K C}(Y), g(0)=I_{0}\right\}$ and introduce the generalized metric $D$ on $E$, which is defined by

$$
\begin{gathered}
D(g, h)=\inf \left\{\mu \in(0, \infty) \mid d_{\infty}(g(x), h(x))\right. \\
\leq \mu \varphi(x, x, x), \forall x \in X\},
\end{gathered}
$$

where, as usual, inf $\emptyset=\infty$. It can easily be verified that $(E, D)$ is a complete generalized metric space (see [25], Theorem 2.4).

We now define the linear mapping $J: E \rightarrow E$ by

$$
J g(x)=2^{-j} g\left(2^{j} x\right)
$$

for all $x \in X$.

Moreover, we can infer from (65) and (66) that

$$
D(f, J f) \leq \begin{cases}\frac{L}{6}, & \text { if } j=-1 \\ \frac{1}{6}, & \text { if } j=1\end{cases}
$$

The rest of the proof is similar to the proof of Theorem 9.

Remark 22. In Theorem 21, if the fuzzy set-valued mapping $f$ degenerates into a set-valued mapping, then the supremum metric $d_{\infty}$ will reduce to the Hausdorff metric $d_{H}$. Thus, this theorem is obviously an extension of Theorems 2.2 and 2.4 in [19].

Corollary 23. Let $j \in\{-1,1\}$ be fixed and let $p, \theta$ be positive real numbers with $p \neq 1$ and let $X$ be a cone with the vertex 
0 contained in a real normed space. Suppose that $f: X \rightarrow$ $\mathscr{F}_{K C}(Y)$ is a mapping satisfying

$$
\begin{aligned}
d_{\infty}( & f\left(\frac{x+y}{2}+z\right) \oplus f\left(\frac{x+z}{2}+y\right) \oplus f\left(\frac{y+z}{2}+x\right), \\
& 2(f(x) \oplus f(y) \oplus f(z))) \\
\leq & \theta\left(\|x\|^{p}+\|y\|^{p}+\|z\|^{p}\right)
\end{aligned}
$$

for all $x, y, z \in X$. Then

$$
A(x)=\lim _{n \rightarrow \infty} 2^{-j n} f\left(2^{j n} x\right)
$$

exists for each $x \in X$ and defines a unique Cauchy-Jensen type additive fuzzy set-valued mapping $A: X \rightarrow \mathscr{F}_{K C}(Y)$ such that

$$
d_{\infty}(f(x), A(x)) \leq \begin{cases}\frac{\theta\|x\|^{p}}{2^{p}-2}, & \text { if } j=-1, p>1, \\ \frac{\theta\|x\|^{p}}{2-2^{p}}, & \text { if } j=1,0<p<1\end{cases}
$$

for all $x \in X$.

Proof. In Theorem 21, let $\varphi(x, y, z)=\theta\left(\|x\|^{p}+\|y\|^{p}+\|z\|^{p}\right)$. Then we can choose $L=2^{j(p-1)}$ and hence we can obtain the desired result.

Corollary 24. Let $j \in\{-1,1\}$ be fixed and let $p, \theta$ be positive real numbers with $p \neq 1 / 3$ and let $X$ be a cone with the vertex 0 contained in a real normed space. Suppose that $f: X \rightarrow$ $\mathscr{F}_{K C}(Y)$ is a mapping satisfying

$$
\begin{gathered}
d_{\infty}\left(f\left(\frac{x+y}{2}+z\right) \oplus f\left(\frac{x+z}{2}+y\right) \oplus f\left(\frac{y+z}{2}+x\right),\right. \\
2(f(x) \oplus f(y) \oplus f(z))) \leq \theta\|x\|^{p}\|y\|^{p}\|z\|^{p}
\end{gathered}
$$

for all $x, y, z \in X$. Then

$$
A(x)=\lim _{n \rightarrow \infty} 2^{-j n} f\left(2^{j n} x\right)
$$

exists for each $x \in X$ and defines a unique Cauchy-Jensen type additive fuzzy set-valued mapping $A: X \rightarrow \mathscr{F}_{K C}(Y)$ such that

$$
d_{\infty}(f(x), A(x)) \leq \begin{cases}\frac{\theta\|x\|^{3 p}}{3\left(2^{3 p}-2\right)}, & \text { if } j=-1, p>\frac{1}{3}, \\ \frac{\theta\|x\|^{3 p}}{3\left(2-2^{3 p}\right)}, & \text { if } j=1,0<p<\frac{1}{3}\end{cases}
$$

for all $x \in X$.

Proof. In Theorem 21, let $\varphi(x, y, z)=\theta\|x\|^{p}\|y\|^{p}\|z\|^{p}$. Then we can choose $L=2^{j(3 p-1)}$ and hence we can obtain the desired result.
Corollary 25. Let $j \in\{-1,1\}$ be fixed and let $p, q, t, \theta$ be positive real numbers with $p+q+t \neq 1$ and let $X$ be a cone with the vertex 0 contained in a real normed space. Suppose that $f: X \rightarrow \mathscr{F}_{K C}(Y)$ is a mapping satisfying

$$
\begin{aligned}
d_{\infty}\left(f\left(\frac{x+y}{2}+z\right) \oplus f\left(\frac{x+z}{2}+y\right) \oplus f\left(\frac{y+z}{2}+x\right),\right. \\
2(f(x) \oplus f(y) \oplus f(z))) \\
\leq \theta\left(\|x\|^{p}\|y\|^{q}\|z\|^{t}+\|x\|^{p+q+t}+\|y\|^{p+q+t}+\|z\|^{p+q+t}\right)
\end{aligned}
$$

for all $x, y, z \in X$. Then

$$
A(x)=\lim _{n \rightarrow \infty} 2^{-j n} f\left(2^{j n} x\right)
$$

exists for each $x \in X$ and defines a unique Cauchy-Jensen type additive fuzzy set-valued mapping $A: X \rightarrow \mathscr{F}_{K C}(Y)$ such that

$$
\begin{aligned}
& d_{\infty}(f(x), A(x)) \\
& \quad \leq \begin{cases}\frac{4 \theta\|x\|^{3 p}}{3\left(2^{p+q+t}-2\right)}, & \text { if } j=-1, p+q+t>1, \\
\frac{4 \theta\|x\|^{p+q+t}}{3\left(2-2^{p+q+t}\right)}, & \text { if } j=1,0<p+q+t<1\end{cases}
\end{aligned}
$$

for all $x \in X$.

Proof. In Theorem 21, let $\varphi(x, y, z)=\theta\left(\|x\|^{p}\|y\|^{q}\|z\|^{t}+\right.$ $\left.\|x\|^{p+q+t}+\|y\|^{p+q+t}+\|z\|^{p+q+t}\right)$. Then we can choose $L=$ $2^{j[(p+q+t)-1]}$ and hence we can obtain the desired result.

\section{Concluding Remark}

In this paper, we proved the Hyers-Ulam-Rassias stability of several types of additive fuzzy set-valued functional equations, including Cauchy, Jensen, and Cauchy-Jensen type fuzzy set-valued functional equations. Our results generalized certain important results obtained by other authors for these equations when they are a single-valued or a set-valued one. Obviously, this paper provided us a novel idea to discuss the stability of functional equations from a more unified perspective. Certainly, further work will focus on the stability of other types of functional equations by using this idea.

\section{Conflict of Interests}

The authors declare that there is no conflict of interests regarding the publication of this paper.

\section{Acknowledgments}

This work was supported by "Qing Lan” Talent Engineering Funds by Tianshui Normal University. Yaoyao Lan acknowledges the support of the National Natural Science Foundation of China (no. 11226268), Scientific and Technological 
Research Program of Chongqing Municipal Education Commission (no. KJ131219), and Program for Innovation Team Building at Institutions of Higher Education in Chongqing (no. KJTD201321). Wei Chen acknowledges the support of the Beijing Municipal Education Commission Foundation of China (no. KM201210038001) and the Humanity and Social Science Youth Foundation of Ministry of Education of China (no. 13YJC630012).

\section{References}

[1] S. M. Ulam, Problems in Modern Mathematics, Wiley, New York, NY, USA, 1960.

[2] D. H. Hyers, "On the stability of the linear functional equation," Proceedings of the National Academy of Sciences of the United States of America, vol. 27, pp. 222-224, 1941.

[3] T. Aoki, "On the stability of the linear transformation in Banach spaces," Journal of the Mathematical Society of Japan, vol. 2, pp. 64-66, 1950.

[4] M. S. Moslehian and T. M. Rassias, "Stability of functional equations in non-Archimedean spaces," Applicable Analysis and Discrete Mathematics, vol. 1, no. 2, pp. 325-334, 2007.

[5] T. M. Rassias, "On the stability of the linear mapping in Banach spaces," Proceedings of the American Mathematical Society, vol. 72, no. 2, pp. 297-300, 1978.

[6] P. Găvruţa, "A generalization of the Hyers-Ulam-Rassias stability of approximately additive mappings," Journal of Mathematical Analysis and Applications, vol. 184, no. 3, pp. 431-436, 1994.

[7] S. M. Jung, Hyers-Ulam-Rassias Stability of Functional Equations in Nonlinear Analysis, Springer, Berlin, Germany, 2011.

[8] V. Radu, "The fixed point alternative and the stability of functional equations," Fixed Point Theory, vol. 4, no. 1, pp. 9196, 2003.

[9] K. Ciepliński, "Applications of fixed point theorems to the Hyers-Ulam stability of functional equations-a survey," Annals of Functional Analysis, vol. 3, no. 1, pp. 151-164, 2012.

[10] A. K. Mirmostafaee and M. S. Moslehian, "Fuzzy versions of Hyers-Ulam-Rassias theorem," Fuzzy Sets and Systems, vol. 159, no. 6, pp. 720-729, 2008.

[11] A. K. Mirmostafaee, M. Mirzavaziri, and M. S. Moslehian, "Fuzzy stability of the Jensen functional equation," Fuzzy Sets and Systems, vol. 159, no. 6, pp. 730-738, 2008.

[12] S. Y. Jang, J. R. Lee, C. Park, and D. Y. Shin, "Fuzzy stability of Jensen-type quadratic functional equations," Abstract and Applied Analysis, vol. 2009, Article ID 535678, 17 pages, 2009.

[13] J. R. Lee, S.-Y. Jang, C. Park, and D. Y. Shin, "Fuzzy stability of quadratic functional equations," Advances in Difference Equations, vol. 2010, Article ID 412160, 16 pages, 2010.

[14] S. A. Mohiuddine and A. Alotaibi, "Fuzzy stability of a cubic functional equation via fixed point technique," Advances in Difference Equations, vol. 2012, p. 48, 2012.

[15] C. Park, "A fixed point approach to the fuzzy stability of an additive-quadratic-cubic functional equation," Fixed Point Theory and Applications, vol. 2009, Article ID 918785, 24 pages, 2009.

[16] K. Nikodem and D. Popa, "On single-valuedness of set-valued maps satisfying linear inclusions," Banach Journal of Mathematical Analysis, vol. 3, no. 1, pp. 44-51, 2009.

[17] G. Lu and C. Park, "Hyers-Ulam stability of additive set-valued functional equations," Applied Mathematics Letters, vol. 24, no. 8, pp. 1312-1316, 2011.
[18] C. Park, D. O’Regan, and R. Saadati, "Stability of some setvalued functional equations," Applied Mathematics Letters. An International Journal of Rapid Publication, vol. 24, no. 11, pp. 1910-1914, 2011.

[19] H. A. Kenary, H. Rezaei, Y. Gheisari, and C. Park, "On the stability of set-valued functional equations with the fixed point alternative," Fixed Point Theory and Applications, vol. 2012, p. 81, 2012.

[20] C. Castaing and M. Valadier, Convex Analysis and Measurable Multifunctions, vol. 580 of Lecture Notes in Mathematics, Springer, Berlin, Germany, 1977.

[21] P. Diamond and P. Kloeden, Metric Spaces of Fuzzy Sets: Theory and Applications, World Scientific, Singapore, 1994.

[22] H. Inoue, "A strong law of large numbers for fuzzy random sets," Fuzzy Sets and Systems, vol. 41, no. 3, pp. 285-291, 1991.

[23] K. Nikodem, K-Convex and K-Concave Set-Valued Functions, Zeszyty Naukowe, Politech, Krakow, Poland, 1989.

[24] J. B. Diaz and B. Margolis, "A fixed point theorem of the alternative, for contractions on a generalized complete metric space," Bulletin of the American Mathematical Society, vol. 74, pp. 305-309, 1968.

[25] M. E. Gordji, C. Park, and M. B. Savadkouhi, "The stability of a quartic type functional equation with the fixed point alternative," Fixed Point Theory, vol. 11, no. 2, pp. 265-272, 2010.

[26] Z. Gajda, "On stability of the Cauchy equation on semigroups," Aequationes Mathematicae, vol. 36, no. 1, pp. 76-79, 1988.

[27] L. Cădariu and V. Radu, "Fixed points and the stability of Jensen's functional equation," Journal of Inequalities in Pure and Applied Mathematics, vol. 4, no. 1, article 4, 7 pages, 2003.

[28] S. M. Jung, "Hyers-Ulam-Rassias stability of Jensen's equation and its application," Proceedings of the American Mathematical Society, vol. 126, no. 11, pp. 3137-3143, 1998. 


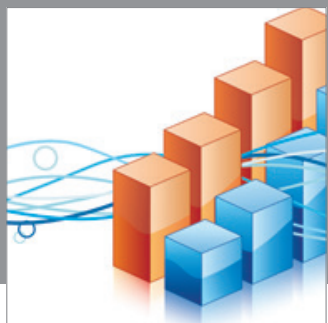

Advances in

Operations Research

mansans

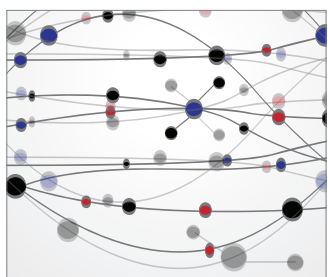

The Scientific World Journal
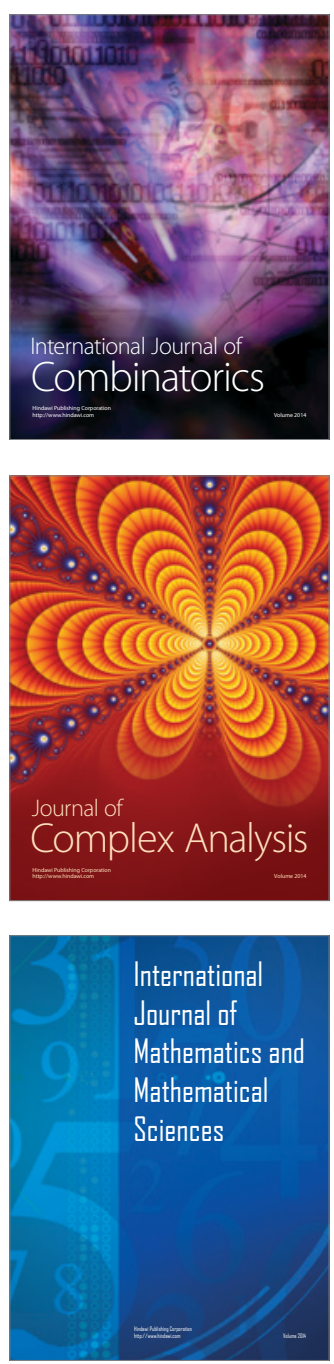
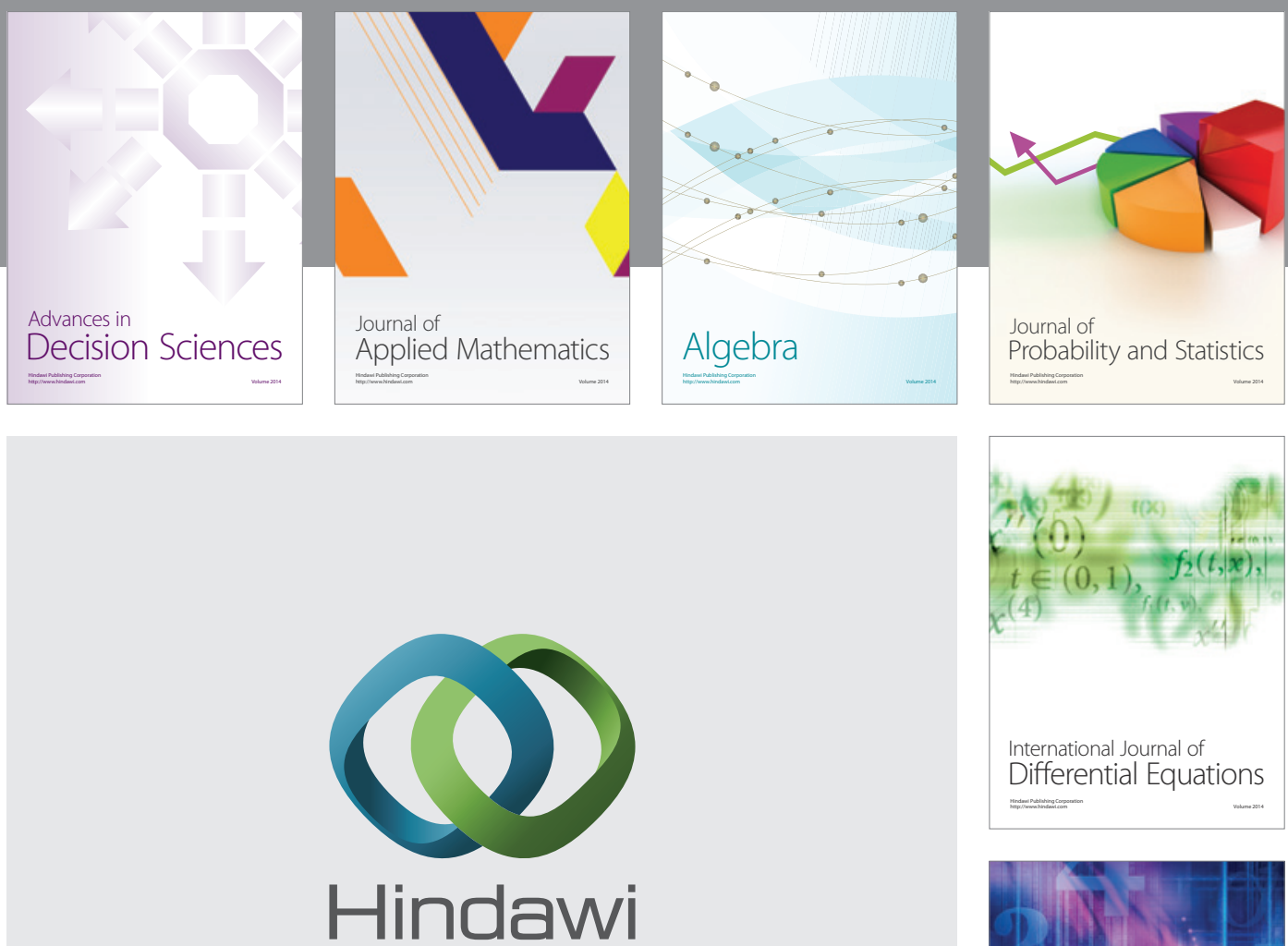

Submit your manuscripts at http://www.hindawi.com
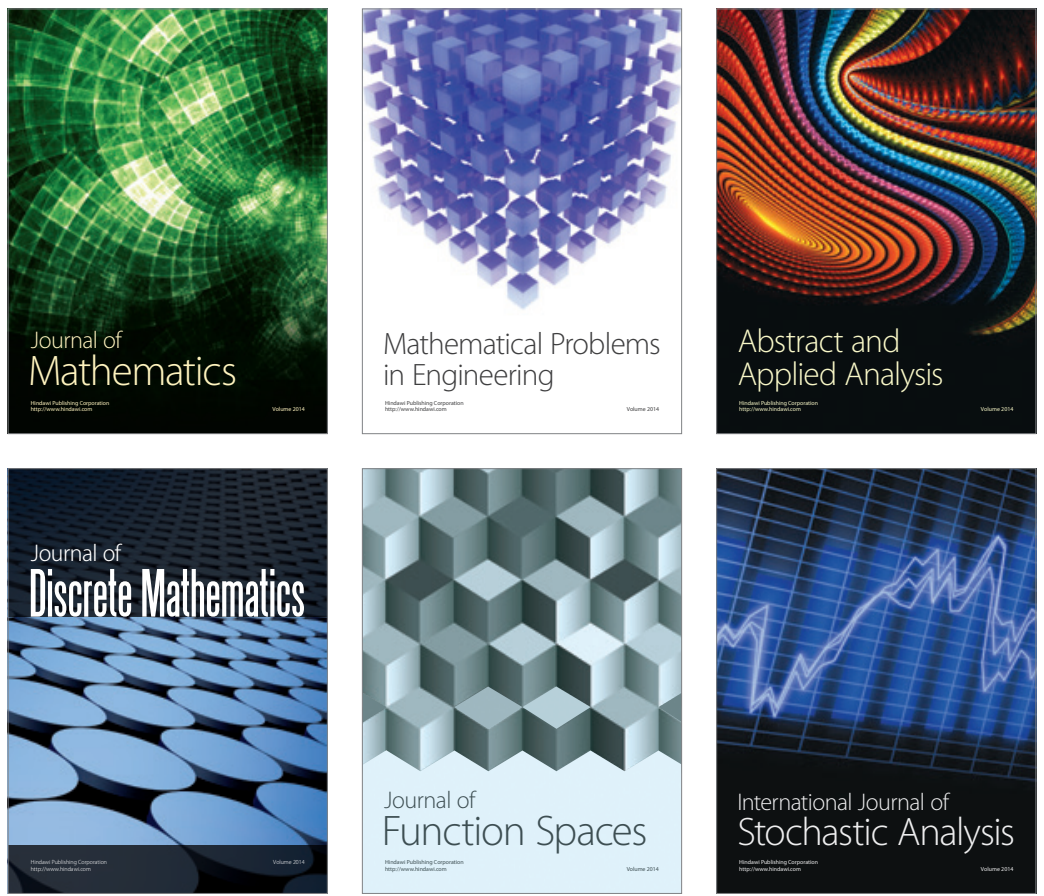

Journal of

Function Spaces

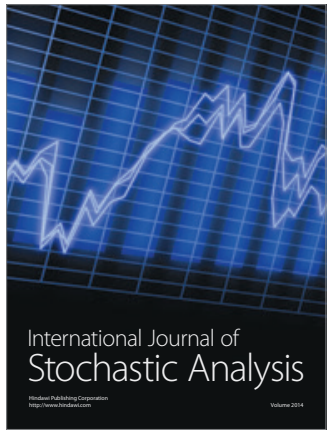

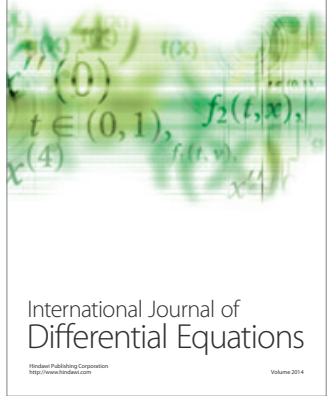
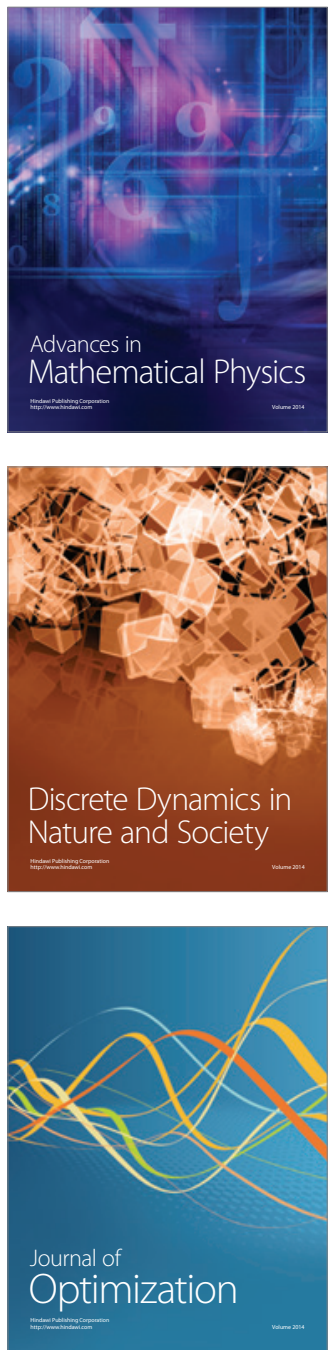